CLINICAL STUDY

\title{
Sexual dimorphism of body composition and insulin sensitivity across pubertal development in obese Caucasian subjects
}

Claudia Brufani, Alberto Tozzi ${ }^{1}$, Danilo Fintini, Paolo Ciampalini, Armando Grossi, Rossana Fiori, Daniela Kiepe, Melania Manco ${ }^{2}$, Riccardo Schiaffini, Ottavia Porzio ${ }^{3}$, Marco Cappa and Fabrizio Barbetti ${ }^{3,4,5}$

Endocrinology and Diabetes Unit, Department of Paediatric Medicine, Bambino Gesù Children's Hospital-IRCCS, Piazza S. Onofrio 4, O0165 Rome, Italy,

${ }^{1}$ Epidemiology Unit, ${ }^{2}$ Scientific Directorate, ${ }^{3}$ Department of Internal Medicine, University of Tor Vergata, Viale Oxford 81, O0134 Rome, Italy,

${ }^{4}$ Laboratory of Molecular Endocrinology and Diabetes, Piazza S. Onofrio 4, 00165 Rome, Italy and ${ }^{5}$ San Raffaele Biomedical Park Foundation, Via di Castel Romano 100, 00134 Rome, Italy

(Correspondence should be addressed to C Brufani; Email: cbrufani@libero.it)

\begin{abstract}
Background: Puberty is a period of rapid growth associated with metabolic, hormonal, and body composition changes that can influence risk factors for chronic diseases such as type 2 diabetes. Objective: To evaluate body composition and insulin sensitivity (IS) modifications throughout puberty in a large group of obese Caucasian subjects.

Methods: Five hundred and nineteen obese subjects (4-19 years), grouped according to gender and Tanner stage $(\mathrm{T})$, underwent oral glucose tolerance test. Quantitative insulin check index (QUICKI) and ISI were calculated as indexes of IS. In 309 subjects, body composition by dual-energy X-ray absorptiometry, IGF1, adiponectin, and leptin were also evaluated.

Results: Body composition modifications were sexually dimorphic, with girls not modifying fat and lean percentage and fat distribution $(P>0.15)$, and boys decreasing fat percentage and increasing lean percentage and central fat depot $(P<0.001)$ across Ts. IS decreased during mid-puberty and returned to prepubertal levels by the end of puberty. Girls showed lower IS than boys $(P<0.01$ and $=0.03$ for QUICKI and ISI respectively). In multivariate analysis factors that negatively influenced IS, independently from $\mathrm{T}$ or age, were total fat mass and central fat depot in girls $(P<0.05$ and $<0.01$, respectively), total fat and lean mass in boys $(P<0.01)$. IGF1, adiponectin, and leptin were not related to pubertal IS.

Conclusions: In obese Caucasian subjects, further decrease of IS observed during puberty is a transient phenomenon. Factors that independently from T or age influence IS are central fat depot in girls, lean amount in boys, and total fat mass in both sexes.
\end{abstract}

European Journal of Endocrinology $160769-775$

\section{Introduction}

As the epidemic of childhood obesity progresses, physicians are increasingly faced with obesity related comorbidities during pubertal transition. Puberty is a dynamic period of development associated with a number of metabolic, hormonal, and body composition changes that can influence risk factors for chronic disease such as type 2 diabetes (1).

Insulin resistance is a feature of obesity and hallmarks normal pubertal development. In normal weight children, insulin sensitivity (IS) is higher before the onset of puberty, reaches its nadir midway through maturation, and approaches near pre-pubertal levels at the end of maturation (2-8). Hormonal changes in the GH/IGF1 axis are thought to be the primary determinants of pubertal insulin resistance. Serum levels of these hormones are higher during puberty than in the prepubertal or adult years, and insulin resistance in adolescents has been shown to positively correlate with serum IGF1 and 24-h GH levels $(4,7)$. However, discordant data have been reported on the insulin resistance pattern during adolescence in obese subjects $(9,10)$.

Puberty is also marked by rapid changes in body size, shape, and composition. During normal pubertal development, there is a significant weight gain: $50 \%$ of adult body weight is gained throughout adolescence (11). Alterations in total adiposity and body fat distribution are thought to be involved in the pubertal insulin resistance, along with the physiological GH hypersecretion (12).

There has been no study to our knowledge that has looked at the relationship between insulin resistance, body composition assessed by direct measure (i.e., dualenergy X-ray absorptiometry; DEXA), and IGF1 in obese Caucasian subjects during pubertal development. 
Therefore, we sought to assess the correlation between the dynamics of IS and the differences in lean and fat mass and the distribution of body fat from childhood to the end of puberty in Italian obese children and adolescents.

\section{Subjects and methods}

We investigated a large group of obese Caucasian children and adolescents (519 subjects), covering a wide range of age (4.0-19.0 years).

Patients referred for obesity from 1997 to 2008, to Bambin Gesù Children's Hospital from their primary care pediatrician were consecutively enrolled into the study, if they met the following criteria: i) age and gender body mass index (BMI; $z$-score) above 2.0 S.D., based on Italian standards (13); ii) absence of underlying chronic diseases; iii) Italian origin (all four grandparents of Italian descent). Participants were not following a weight-reducing diet, not taking any medication or carrying a previous clinical diagnosis known to influence body composition, IS or dietary intake. Written informed consent was obtained from parents or patients before any testing procedure. Ethical approval of the protocol was obtained by the Local Scientific Committee. The study was conducted in accordance with the Declaration of Helsinki.

After a $12 \mathrm{~h}$ overnight fast, at $\sim 0800 \mathrm{~h}$ all subjects were admitted in the clinic for 1-day inpatient visit. Height was measured without shoes to the nearest $0.1 \mathrm{~cm}$ using a wall stadiometer, and weight was measured in underwear to the nearest $0.1 \mathrm{~kg}$ using a medical balance beam scale. Physical maturation was assessed according to Marshall and Tanner (14). Subjects were divided into gender and Tanner stage $(\mathrm{T})$ on the basis of breast development in girls, genitalia development in boys, as follows: prepubertal (T1), early pubertal (T2), mid-pubertal (T3-4), and late pubertal (T5).

\section{Insulin sensitivity indexes}

Two baseline fasting blood samples were taken via antecubital vein catheter and then all subjects underwent an oral glucose tolerance test (OGTT; $1.75 \mathrm{~g}$ of glucose solution per kilogram of body weight to a maximum of $75 \mathrm{~g}$ ). Plasma samples were collected every 30 min until $2 \mathrm{~h}$, for determination of glucose and insulin concentration.

IS was estimated by quantitative insulin check index (QUICKI) (15) based on fasting glucose and insulin values and by IS index (ISI) (16), an OGTT derived index. These indexes have been demonstrated to strongly correlate with the euglycemic-hyperinsulinemic clamp and minimal model frequently sampled i.v. glucose tolerance test derived measures of IS in obese children and adolescents (17-19).

\section{Body composition, IGF1, adiponectin, and leptin}

We added body composition evaluation to our study protocol in 2002 and assessed it in 309 subjects (145 girls, 164 boys). In this subgroup, IGF1, adiponectin, and leptin were also measured.

Body composition was measured by DEXA using Hologic QDR Delphi (Hologic Inc., Bedford, MA, USA). DEXA segments the body into the three compartments of fat mass, bone mineral content, and fat-free soft tissue, i.e., lean mass. DEXA data consisted of fat mass $(\mathrm{kg})$, lean mass $(\mathrm{kg})$, and bone mineral mass $(\mathrm{kg})$ of the trunk, upper, and lower extremities, and head. The trunk region was defined as the region below the chin, the region delineated by vertical lines within the left and right glenoid fossa and bordering laterally to the ribs, and the region delineated by oblique lines that cross the femoral necks and converge below the pubic symphysis. Body fat distribution was evaluated by central obesity index as the fat mass in the truncal region divided by the fat mass in the lower extremity region (20).

\section{Assays}

Serum insulin levels were measured by a chemiluminescent immunoassay method on ADVIA Centaur analyzer with a commercial kit (ADVIA Centaur IRI); intra- and inter-assay CV range was 3.3-4.6 and 2.6$5.9 \%$ respectively. Quantitative determinations of blood glucose were measured by enzymatic method on Roche automated clinical chemistry analyzer (Roche/Hitachi 904 analyzer); intra- and inter-assay CV were 0.9 and $1.8 \%$ respectively. IGF1 levels were measured by IRMA (Immunotech, Beckman Coulter Laboratories, SA, Marseille Cedex, France) according to the manufacturer's instructions. Human adiponectin was determined in serum by ELISA (Biovendor Laboratory Medicine Inc., Brno, Czech Republic) according to the manufacturer's instructions. Human leptin was determined in serum by IRMA (DSL, Beckman Coulter, Webster, TX, USA) according to the manufacturer's instructions.

\section{Statistical analysis}

Means and S.D. values were computed within sex and T categories, unless otherwise noted. Girls and boys were analyzed separately due to the differences in hormone and body composition modifications during puberty. When the variable of interest was non-normally distributed, parametric analyses on a suitable transformed variable were performed.

Two statistical procedures were used when looking for differences on characteristics within and across gender and $\mathrm{T}$ : i) two sample $t$-test for comparison between two groups; ii) one-way ANOVA with four groups (T1, T2, T3-4, T5) to compare characteristics across these four. For post hoc multiple comparison, 
we utilized Bonferroni's test. Proportions were compared by $\chi^{2}$ test.

Stepwise multiple linear regression analysis was performed by entering closely interrelated variables separately, i.e., when lean mass was inserted, fat mass was omitted, because fat and lean mass were highly and positively correlated. ISI was the dependent variables. Significance level for all tests was set at $P<0.05$. SPSS software, version 12.0 (SPSS Inc., Chicago, IL, USA) was used for all analyses.

\section{Results}

Characteristics of subjects divided according to gender and $\mathrm{T}$ are reported in Table 1. Participants included 228 girls and 291 boys. Girls were younger than boys $(P=0.02)$, due to the later puberty of boys. Height and BMI increased throughout puberty, with the greatest increment in height occurring from $\mathrm{T} 1$ to $\mathrm{T} 2$ in girls and from $\mathrm{T} 2$ to $\mathrm{T} 3-4$ in boys, and in BMI from $\mathrm{T} 2$ to T3-4 in both. There was no significant difference in BMI between boys and girls at any $\mathrm{T}$.

The 309 subjects with body composition, IGF1, adiponectin, and leptin assessment were equally distributed in the different Ts and were comparable in terms of BMI, age, and IS with other subjects included in the study.

\section{Body composition throughout puberty}

Both girls and boys accrued fat mass at a similar rate: at the end of puberty, the average difference in fat mass was $+12 \mathrm{~kg}$ for girls $(+53 \%),+9 \mathrm{~kg}$ for boys $(+39 \%)$ $(P=0.53)$. Boys appeared to have a more pronounced augment in lean mass than girls; at the end of puberty, the average increase was $18 \mathrm{~kg}$ for girls $(+62 \%)$ and $29 \mathrm{~kg}$ for boys $(+92 \%)$, but the difference was not statistically significant $(P=0.28)$.

When percentages of total body fat and lean mass were considered, girls showed steady body composition throughout puberty $(P=0.15$ and 0.24 for fat and lean percentage respectively), not modifying their lean to fat ratio. On the contrary, in boys, total body lean percentage increased and conversely fat percentage decreased from T3-4 onwards $(P<0.001$ for T3-4 vs
$\mathrm{T} 1$, and for T5 vs T1, for both lean and fat percentages). Girls had higher total body fat percentage and lower lean percentage than boys at each $\mathrm{T}(P<0.01$; Fig. 1$)$.

When body fat distribution was considered, girls showed constant values of central obesity index across Ts $(P=0.61)$. By contrast, central obesity index greatly increased at $\mathrm{T} 5$ in boys $(P<0.001$ for $\mathrm{T} 5$ vs T1; $P=0.04$ vs $\mathrm{T} 5$ of girls; Fig. 2).

Furthermore, to evaluate any modification of body composition after menarche, girls with T3-4 and T5 were divided according to the presence/absence of menarche. There were no differences in fat and lean percentage and central obesity index between these two groups $(P=0.96,0.90,0.86$ respectively $)$.

\section{Insulin sensitivity, IGF1, adiponectin, and leptin throughout puberty}

ISI and QUICKI were highly correlated in girls and boys $(r=0.90, P<0.001)$.

QUICKI decreased with the onset of puberty, reaching the lowest values in mid-puberty, at T3-4 $(P<0.001$ vs $\mathrm{T} 1$ ), and returning to almost the prepubertal levels at T5, in both girls and boys. As a whole group, girls had lower QUICKI values than boys $(P=0.001)$, and lower QUICKI than boys at each T. However, statistical significance was only achieved for $\mathrm{T} 2 \quad(P=0.03$; Fig. 3A).

ISI showed similar findings, with IS decreasing from T2 in girls, $(P=0.02$ vs T1), and from T3-4 in boys, $(P=0.01$ vs $\mathrm{T} 1)$ and recovering by $\mathrm{T} 5$ in both sexes. Again, girls as a group had lower ISI values than boys $(P=0.03)$ and lower ISI than boys at each $\mathrm{T}$, but statistical significance was only achieved for $\mathrm{T} 2$ $(P=0.02$; Fig. 3B $)$.

IGF1 increased in boys and girls at T3-4 $(P<0.001$ vs T1) (mean IGF1 at T1 and T3-4 241 133 and $468 \pm 185 \mathrm{ng} / \mathrm{ml}$ respectively) and maintained high values at $\mathrm{T} 5(468 \pm 174 \mathrm{ng} / \mathrm{ml})$.

Adiponectin and leptin did not show great variation during puberty, even if lower value of adiponectin at $\mathrm{T} 3-4$ in boys $(10.8 \pm 4.9 \mu \mathrm{g} / \mathrm{ml})$ vs $\mathrm{T} 1$ $(14.2 \pm 5.1 \mu \mathrm{g} / \mathrm{ml} ; P=0.011)$, and higher values of leptin in girls at $\mathrm{T} 5(7.3 \pm 2.2 \mathrm{ng} / \mathrm{ml})$ vs $\mathrm{T} 1(5.8 \pm 1.9$; $P=0.007)$ were observed.

Table 1 General characteristics of 519 obese subjects according to gender and pubertal stage.

\begin{tabular}{|c|c|c|c|c|c|c|c|c|}
\hline & \multicolumn{2}{|c|}{ T1 } & \multicolumn{2}{|c|}{ T2 } & \multicolumn{2}{|c|}{ T3-4 } & \multicolumn{2}{|c|}{ T5 } \\
\hline & $\begin{array}{c}\text { Girls } \\
(n=65)\end{array}$ & $\begin{array}{c}\text { Boys } \\
(n=103)\end{array}$ & $\begin{array}{c}\text { Girls } \\
(n=41)\end{array}$ & $\begin{array}{c}\text { Boys } \\
(n=87)\end{array}$ & $\begin{array}{c}\text { Girls } \\
(n=69)\end{array}$ & $\begin{array}{c}\text { Boys } \\
(n=72)\end{array}$ & $\begin{array}{c}\text { Girls } \\
(n=53)\end{array}$ & $\begin{array}{c}\text { Boys } \\
(n=29)\end{array}$ \\
\hline Age (years) & $8.6 \pm 1.7$ & $9.7 \pm 1.8$ & $10.5 \pm 1.4$ & $12.0 \pm 1.4$ & $12.7 \pm 1.7$ & $14.1 \pm 1.4$ & $14.4 \pm 2.6$ & $16.0 \pm 1.6$ \\
\hline Height $(\mathrm{cm})$ & $135.0 \pm 9.8$ & $142.7 \pm 10.4$ & $147.3 \pm 8.3$ & $154.5 \pm 8.8$ & $156.0 \pm 7.2$ & $168.0 \pm 8.0$ & $160.5 \pm 5.7$ & $173.7 \pm 7.2$ \\
\hline Weight (kg) & $51.1 \pm 11.4$ & $56.7 \pm 11.0$ & $63.0 \pm 12.6$ & $69.7 \pm 16.5$ & $78.7 \pm 16.4$ & $89.2 \pm 17.2$ & $87.0 \pm 15.2$ & $99.1 \pm 13.7$ \\
\hline $\operatorname{BMI}\left(\mathrm{kg} / \mathrm{m}^{2}\right)$ & $27.8 \pm 3.9$ & $27.6 \pm 3.3$ & $28.8 \pm 3.5$ & $28.9 \pm 4.4$ & $32.2 \pm 5.3$ & $31.5 \pm 4.5$ & $33.8 \pm 5.6$ & $32.9 \pm 4.9$ \\
\hline
\end{tabular}

T: Tanner stage. 

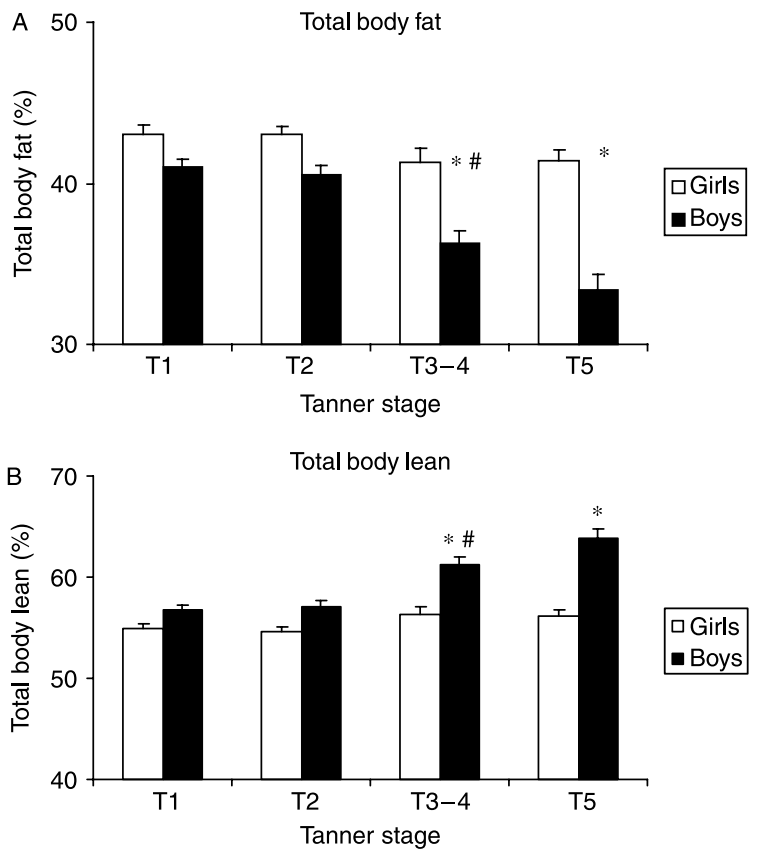

Figure 1 (A) Total body fat (\%) by Tanner stage and sex. ${ }^{*} P<0.05$ compared with T1 of the same gender; \#P<0.05 compared with preceding Tanner stage of the same gender. Data are expressed as means \pm S.E.M. The group of 145 girls had higher fat mass percentage than the group of 164 boys $(P<0.001)$. Girls had higher fat mass percentage than boys at each Tanner stage $(P<0.01)$. Two-sample $t$-test for comparison between girls and boys and one-way ANOVA with four groups (T1, T2, T3-4, T5) to compare characteristics across these four, were used. (B) Total body lean (\%) by Tanner stage and sex. ${ }^{*} P<0.05$ compared with $\mathrm{T} 1$ of the same gender; $\# P<0.05$ compared with preceding Tanner stage of the same gender. Boys (164 subjects) had higher lean mass percentage than girls (145 subjects) $(P<0.001)$. Boys had higher lean mass percentage than girls at each Tanner stage $(P<0.01)$. Statistical methods as in A were applied.

\section{Prediction of IS}

In multivariate analysis with IS indexes as dependent variables, parameters independently associated to IS were central obesity index and total body fat mass $(P<0.01$ and $<0.05$ respectively $)$ in girls; total body fat and lean mass $(P<0.01)$ in boys (Table 2$)$. These relationships were independent of T, IGF1, adiponectin, and leptin. When age was inserted as covariate instead of T, the relationship between IS and body composition did not change, and age was not significantly linked to IS.

\section{Discussion}

This cross-sectional analysis of 519 children and adolescents (309 with body composition evaluation) represents the largest examination to date of relation of the IS to Tanner stage, sex, and body composition in obese subjects.

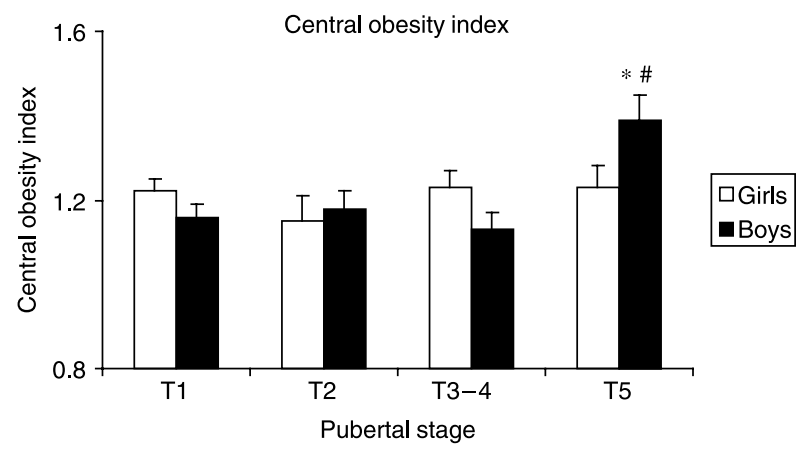

Figure 2 Central obesity index by Tanner stage and sex. ${ }^{\star} P<0.05$ compared with T1 of the same gender; \#P<0.05 compared with preceding Tanner stage of the same gender. Central obesity index was not different between the group of 164 boys and the group of 145 girls. Boys had higher central obesity index than girls at T5 $(P=0.04)$. Statistical methods as in Figure 1.

Data from the present study clearly show that girls are more insulin resistant than boys and that IS decreases by $\mathrm{T} 2$ in girls and by $\mathrm{T} 3-4$ in boys, and returns to $\mathrm{T} 1$ levels by the end of puberty (T5) in both girls and boys. Moreover, body composition modifications are sexually dimorphic, with girls not modifying their fat and lean percentage and their fat distribution during puberty, and boys decreasing fat percentage and increasing lean percentage and central fat depot with age. These changes do not parallel the transient pubertal modification of IS, suggesting that changes in body composition are not the driving force behind the insulin resistance of puberty in obese subjects. In multivariate analysis, factors that primarily influenced IS independent of T or age, are central fat depot in girls, lean mass in boys, and total body fat in both.

In normal weight children, pubertal decrease in IS is a transient physiological stage of development (11). Our findings regarding pubertal decrease of IS in obese subjects are in complete agreement with those of previous cross-sectional and longitudinal observations on normal weight children $(2,3,5)$. The largest crosssectional study was reported in data from 357 black and white children in whom insulin action was assessed by euglycemic clamp technique. In this study, IS began to fall by $\mathrm{T} 2$, reached a nadir at $\mathrm{T} 3$, and then recovered to near prepubertal levels by T5 (2).

Significant change in body composition is known to occur during puberty in concert with changes in the hormonal environment. In normal weight subjects, body fat percent increases in girls and decreases in boys. Total fat mass increases in both sexes, however, because there is a dramatic increase in fat-free mass in boys, their fat mass contributes proportionally less to body weight (11).

In the present study, boys, as expected, accrued more lean than fat mass and at the end of pubertal development, had an increase in lean and fat mass of 92 and 39\% respectively. Girls accrued lean and fat at a 

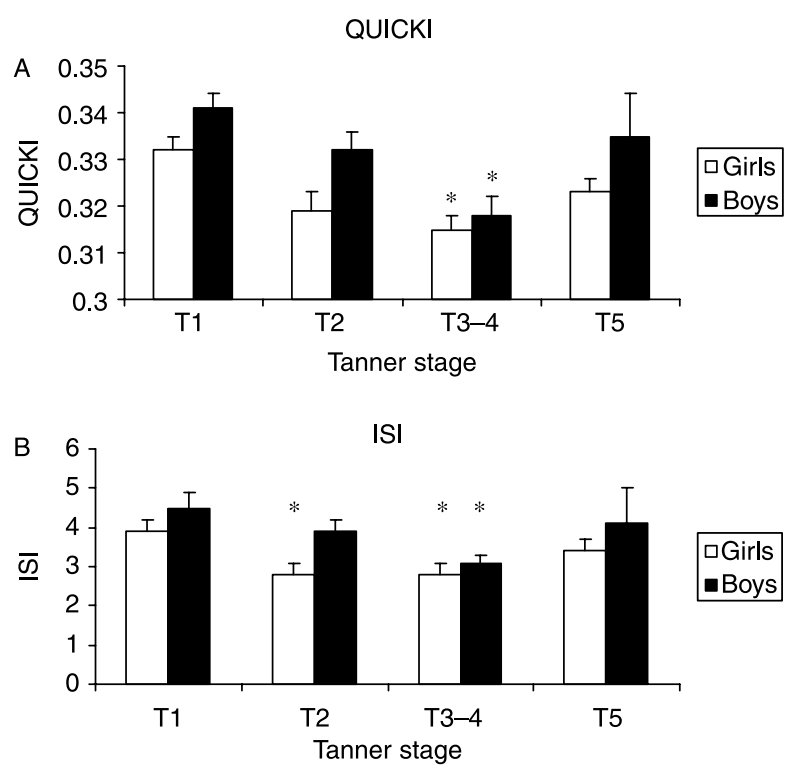

Figure 3 (A) QUICKI (insulin sensitivity index derived from fasting values) by Tanner stage and sex. Data are expressed as means \pm S.E.M. ${ }^{*} P<0.05$ compared with $\mathrm{T} 1$ of the same gender. As a group, girls showed lower QUICKI values than boys $(P=0.001)$. Girls appeared to have lower QUICKI than boys at each Tanner stage, but only differences at T2 were statistically significant $(P=0.03)$. Statistical methods utilized for comparison between girls and boys and across T stages as in Figure 1. (B) ISI (insulin sensitivity index derived from oral glucose tolerance test values) by Tanner stage and sex. ${ }^{*} P<0.05$ compared with $\mathrm{T} 1$ of the same gender. In general, girls had lower ISI values than boys $(P=0.03)$. Girls appeared to have lower ISI than boys at each Tanner stage, but statistical significance was only achieved for T2 $(P=0.02)$.

similar rate (62 and 53\% respectively). Thus, differently from normal weight adolescents, girls maintained a constant body composition, with no body fat percentage increase across pubertal development. Boys emerged from puberty with higher central body fat depot and girls with higher peripheral body fat (i.e. lower body fat; data not shown). The deposition and distribution of fat as these subjects become adults is of importance because accumulating greater amounts of truncal fat may lead them to unfavorable cardiovascular risk status. These findings, in agreement with other authors (21), raise the possibility that women in general have a fat-storage capacity in peripheral adipose tissue exceeding that of men, in whom central fat depot is increased.

In normal weight subjects, substantial evidence suggests that GH may play a key role in pubertal insulin resistance (22). This hypothesis is attractive because GH and IGF1 are transiently higher in mid-puberty, mirroring the changes in IS, and IS is correlated with GH and IGF1 $(4,7)$. Furthermore, the pubertal IS nadir occurs at the same time as the growth spurt. By contrast, sexual steroids do not seem to play a role in physiological changes of IS during pubertal development (23).

Our auxological data support the relationship between pubertal insulin resistance and growth, since IS nadir in our patients was temporally related to the pubertal growth spurt in each sex, at T2 in girls, at T3-4 in boys. Apparently, IGF1 in obese subjects is not a valid tool to monitor growth, as adolescent obesity is associated with profound disturbance in the GH-IGF-IGFBP system (24). In accordance with this view, in the present study, IGF1 was neither inversely correlated with pubertal IS nor temporally related to growth spurt.

In our large group of obese children and adolescents, factors that significantly influenced IS in girls independently from $\mathrm{T}$ or age were total fat mass and central fat depot. Hence, adiposity in girls and specifically central (truncal) obesity has a major role in predicting low IS. While central obesity in growing boys appears to be linked to age (i.e., truncal obesity emerges at T5), in girls central adiposity seems to be strictly related to adverse metabolic outcomes since childhood. This is in accordance with studies on obese girls with polycystic ovary syndrome (PCOS), who are more likely to have abdominal distribution of body fat, which is associated with insulin resistance and hyperandrogenaemia $(25,26)$. However, in the present study, we did not

Table 2 Multiple regression analysis of insulin sensitivity in girls and boys.

\begin{tabular}{|c|c|c|c|c|c|c|c|c|}
\hline \multirow[b]{4}{*}{ Independent variables } & \multicolumn{8}{|c|}{ Dependent variables } \\
\hline & \multicolumn{4}{|c|}{ Girls } & \multicolumn{4}{|c|}{ Boys } \\
\hline & \multicolumn{2}{|c|}{ QUICKI } & \multicolumn{2}{|c|}{ ISI } & \multicolumn{2}{|c|}{ QUICKI } & \multicolumn{2}{|l|}{ ISI } \\
\hline & B coefficient & $\mathrm{P}$ & B coefficient & $P$ & B coefficient & $P$ & B coefficient & $P$ \\
\hline Pubertal stage & -0.06 & 0.53 & -0.13 & 0.22 & -0.03 & 0.79 & -0.13 & 0.28 \\
\hline Central obesity index & -0.33 & $<0.01$ & -0.41 & $<0.01$ & -0.01 & 0.89 & -0.18 & 0.07 \\
\hline Total body fat mass $(\mathrm{kg})$ & -0.19 & 0.05 & -0.24 & 0.02 & -0.42 & $<0.01$ & -0.45 & $<0.01$ \\
\hline Total body fat (\%) & -0.13 & 0.20 & -0.13 & 0.20 & 0.01 & 0.99 & 0.11 & 0.30 \\
\hline Total body lean mass $(\mathrm{kg})$ & -0.11 & 0.28 & -0.17 & 0.11 & -0.31 & $<0.01$ & -0.42 & $<0.01$ \\
\hline Total body lean (\%) & 0.12 & 0.22 & 0.13 & 0.20 & -0.01 & 0.92 & -0.13 & 0.24 \\
\hline IGF1 (ng/ml) & -0.15 & 0.13 & -0.16 & 0.12 & 0.04 & 0.66 & 0.01 & 0.18 \\
\hline Adiponectin $(\mu \mathrm{g} / \mathrm{ml})$ & 0.09 & 0.93 & 0.08 & 0.45 & 0.05 & 0.56 & 0.06 & 0.60 \\
\hline Leptin (ng/ml) & -0.19 & 0.06 & -0.15 & 0.16 & -0.09 & 0.39 & -0.07 & 0.53 \\
\hline
\end{tabular}


investigate the role of PCOS, as most of the girls were premenarcheal or in the first two years after menarche.

Surprisingly, in boys lean mass along with fat mass amount, and independently from $\mathrm{T}$ or age, negatively influenced IS. Our data show for the first time that, contrary to the general belief, higher level of lean body mass is associated with low IS in growing obese male subjects. Many studies on children and adolescents have reported the relationship between body fat and IS $(2,3$, $12,27)$, but no one has reported the relation among IS and lean mass. This could be due to the fact that many studies focused on degree of adiposity, directly measured or estimated by BMI, without taking into account lean mass. One can hypothesize that different muscle fibers distribution and composition in growing boys and girls, with fast fibers closely related to insulin resistance occupying a greater area in men and slow fibers occupying a greater area in women could explain the strict relationship between lean mass and IS found in boys and not in girls (28-29). Our observation must be interpreted with caution since studies have shown that resistance training programs aimed to increase muscle mass improve IS $(30,31)$. Thus, further studies are needed to confirm and explain the link between decreased IS and lean mass we found.

Some limits of our study must be taken into account. First, we used DEXA that can not distinguish visceral from subcutaneous fat, but barely assesses the amount of fat in the trunk. Secondly, we did not study IS with the gold standard technique, i.e., hyperinsulinemiceuglycemic clamp, for its complexity in pediatric population. Finally, cause-and-effect relationships cannot be drawn given the cross-sectional study design. To this purpose, follow-up longitudinal analysis will be performed as this cohort progresses through puberty. Despite these limitations, our investigation provides some robust cross-sectional data on metabolic and anthropometric characteristics of obese Caucasian individuals during pubertal development.

In conclusion, obese children, like normal weight children, experience a stage of decreased IS during puberty. This corresponds with the period of rapid growth and development and resolves by the end of puberty. Total body fat amount in both girls and boys, central body fat depot in girls, and lean mass in boys are the main determinates of IS in growing obese subjects.

\section{Declaration of interest}

There is no conflict of interest that could be perceived as prejudicing the impartiality of the research reported.

\section{Funding}

This research was funded by Ministry of Research, PRIN 2006 to F. Barbetti Research Unit.

\section{Acknowledgements}

We thank Mrs Rita Perinelli, Mrs Leonarda Piras, Mrs Silvia Tiozzo, Mrs Marina Ceccarelli, and Mrs Francesca Romana Garofolo from the nurse staff and Mrs Luciana Luciani, secretary of the Endocrinology and Diabetes Unit, for their assistance in this research project. The authors would also like to thank all the children and families who participated in this study.

\section{References}

1 Siervogel RM, Demerath EW, Schubert C, Remsberg KE, Chumlea WC, Sun S, Czerwinski SA \& Towne B. Puberty and body composition. Hormone Research $20006036-45$.

2 Moran A, Jacobs DR Jr, Steinberger J, Hong CP, Prineas R, Luepker R \& Sinaiko AR. Insulin resistance during puberty: results from clamp studies in 357 children. Diabetes $1999 \mathbf{4 8}$ 2039-2044.

3 Goran MI \& Gower BA. Longitudinal study on pubertal insulin resistance. Diabetes $2001502444-2450$.

4 Amiel SA, Sherwin RS, Simonson DC, Lauritano AA \& Tamborlane WV. Impaired insulin action in puberty: a contributing factor to poor glycemic control in adolescents with diabetes. New England Journal of Medicine 1986315 215-219.

5 Hannon TS, Janosky J \& Arslanian SA. Longitudinal study of physiologic insulin resistance and metabolic changes of puberty. Pediatric Research 200660 759-763.

6 Caprio S, Plewe G, Diamond MP, Simonson DC, Boulware SD, Shervin RS \& Tamborlane WV. Increased insulin secretion in puberty: a compensatory response to reductions in insulin sensitivity. Journal of Pediatrics 1989114 963-967.

7 Moran A, Jacobs DR, Steinberger J, Cohen P, Hong CP, Prineas R \& Sinaiko A. Association between the insulin resistance of puberty and the insulin-like growth factor-I/growth hormone axis. Journal of Clinical Endocrinology and Metabolism $2002 \mathbf{8 7}$ $4817-4820$.

8 Hoffaman RP, Vicini P, Sivitz WI \& Cobelli C. Pubertal adolescent male-female differences in insulin sensitivity and glucose effectiveness determined by the one compartment minimal model. Pediatric Research $2000 \mathbf{4 8} 384-388$.

9 Guzzaloni G, Grugni G, Mazzilli G, Moro D \& Morabito F. Comparison between $\beta$-cell function and insulin resistance indexes in prepubertal and pubertal obese children. Metabolism 200251 1011-1016.

10 Ball GDC, Weigensberg MJ, Cruz ML, Shaibi GQ, Kobaissi HA \& Goran M. Insulin sensitivity, insulin secretion and $\beta$-cell function during puberty in overweight Hispanic children with a family history of type 2 diabetes. International Journal of Obesity 200529 1471-1477.

11 Rogol AD, Roemmich JN \& Clark PA. Growth at puberty. Journal of Adolescent Health 200231 192-200.

12 Roemmich JN, Clark PA, Lusk M, Friel A, Weltman A, Epstein LH \& Rogol AD. Pubertal alterations in growth and body composition. VI. Pubertal insulin resistance: relation to adiposity, body fat distribution and hormone release. International Journal of Obesity 200226 701-709.

13 Luciano A, Bressan F \& Zoppi G. Body mass index reference curves for children aged 3-19 years from Verona, Italy. European Journal of Clinical Nutrition 199751 6-10.

14 Tanner JM. Growth and maturation during adolescence. Nutrition Reviews $19813943-55$.

15 Katz A, Nambi SS, Mather K, Baron AD, Follmann DA, Sullivan G \& Quon MJ. Quantitative insulin sensitivity check index: a simple, accurate method for assessing insulin sensitivity in humans. Journal of Clinical Endocrinology and Metabolism 200085 24022410.

16 Matsuda M \& DeFronzo RA. Insulin sensitivity indices obtained from oral glucose tolerance testing: comparison with the euglycemic insulin clamp. Diabetes Care 199922 1462-1470. 
17 Conwell LS, Brown WJ, Trost SG, Brown W \& Batch J. Indexes of insulin resistance and secretion in obese children and adolescents. Diabetes Care 200427 314-319.

18 Yeckel CW, Weiss R, Dziura J, Taksali SE, Dufour S, Burgert TS, Tamborlane WV \& Caprio S. Validation of insulin sensitivity indices from oral glucose tolerance test parameters in obese children and adolescents. Journal of Clinical Endocrinology and Metabolism 200489 1096-1101.

19 Gungor N, Saad R, Janosky J \& Arslanian S. Validation of surrogate estimates of insulin sensitivity and insulin secretion in children and adolescents. Journal of Pediatrics 2004144 47-55.

20 Daniels SR, Morrison JA, Sprecher DL, Khoury P \& Kimball TR. Association of body fat distribution and cardiovascular risk factors in children and adolescents. Circulation 199999 541-545.

21 Vega GL, Adams-Huet B, Peshock R, Willet DW, Shah B \& Grundy SM. Influence of body fat content and distribution on variation in metabolic risk. Journal of Clinical Endocrinology and Metabolism 200691 4459-4466.

22 Goran MI, Ball GDC \& Cruz ML. Obesity and risk of type 2 diabetes and cardiovascular disease in children and adolescents. Journal of Clinical Endocrinology and Metabolism 200388 1417-1427.

23 Goran MI, Ball GD \& Cruz ML. Obesity and risk of type 2 diabetes and cardiovascular disease in children and adolescents. Journal of Clinical Endocrinology and Metabolism 200388 1417-1427.

24 Dunger DB. Effects of obesity on growth and puberty. Best Practice and Research. Clinical Endocrinology and Metabolism 2005 19 375-390.
25 Diamanti-Kandarakis E. Role of obesity and adiposity in polycystic ovary syndrome. International Journal of Obesity 2007 31 S8-S13.

26 Tfayli $\mathrm{H} \&$ Arslanian S. Menstrual health and the metabolic syndrome in adolescents. Annals of the New York Academy of Sciences 20081135 85-94.

27 Travers SH, Barrett WJ, Bloch CA, Hill JO \& Eckel RH. Gender and Tanner stage differences in body composition and insulin sensitivity in early pubertal children. Journal of Clinical Endocrinology and Metabolism $1995 \mathbf{8 0} 172-178$.

28 Staron RS, Hagerman FC, Hikida RS, Murray TF, Hostler DP, Crill MT, Ragg KE \& Toma K. Fiber type composition of the vastus lateralis muscle of young men and women. Journal of Histochemistry and Cytochemistry $2000 \mathbf{4 8} 623-629$.

29 Lillioja S, Young AA, Culter CL, Ivy JL, Abbott WG, Zawadzki JK, Yki H, Christin L, Secomb TW \& Bogardus C. Skeletal muscle capillary density and fiber type are possible determinants of in vivo insulin resistance in man. Journal of Clinical Invesigation $1987 \mathbf{8 0}$ 415-424.

30 Eriksson JG. Exercise and the treatment of type 2 diabetes mellitus. An update. Sports Medicine 199927 381-391.

31 Andersen JL, Schjerling P, Andersen LL \& Dela F. Resistance training and insulin action in humans: effects of de-training. Journal of Physiology 2003551 1049-1058.

Received 12 February 2009

Accepted 16 February 2009 